\title{
Rechtsprechung
}

\section{Herausgabeanspruch nach §985 BGB trotz der besonderen Regelungen über die Wiedergutmachung des nationalsozialistischen Unrechts}

Bundesgerichtshof, Urteil vom 16. März 2012 - V ZR 279/10

Die Rückerstattungsanordnung für das Land Berlin schließt den Herausgabeanspruch nach §985 BGB nicht aus, wenn der verfolgungsbedingt entzogene Vermögensgegenstand nach dem Krieg verschollen war und der Eigentümer erst nach Ablauf der Frist für die Anmeldung eines Rückerstattungsanspruchs von seinem Verbleib Kenntnis erlangt hat.

\section{Tenor}

- Auf die Revision des Klägers und unter Zurückweisung der Anschlussrevision der Beklagten wird das Urteil des 8. Zivilsenats des Kammergerichts in Berlin vom 28. Januar 2010 im Kostenpunkt und insoweit aufgehoben, als die Klage auf Herausgabe des Plakats "Dogge" abgewiesen und der Widerklage stattgegeben worden ist.

Im Umfang der Aufhebung wird die Berufung der Beklagten gegen das Urteil der Zivilkammer 19 des Landgerichts Berlin vom 10. Februar 2009 zurückgewiesen. Die weitergehende Berufung bleibt zurückgewiesen.

Die Kosten der Rechtsmittelverfahren trägt die Beklagte.

\section{Tatbestand}

Der Kläger ist der Sohn und Rechtsnachfolger von Dr. Hans Sachs. Dieser hatte seit 1896 eine umfangreiche und wertvolle Plakatsammlung zusammengetragen, welche ihm 1938 im Auftrag des Reichspropagandaministeriums weggenommen wurde. Dr. Sachs verließ Deutschland wegen der nationalsozialistischen Judenverfolgung Ende 1938 und emigrierte in die USA.

Nach dem Krieg war die Plakatsammlung zunächst verschollen. Im Jahr 1961 erhielt Dr. Sachs aufgrund eines in einem Verfahren nach dem Bundesrückerstattungsgesetz geschlossenen Vergleichs 225.000 DM als Wiedergutmachung für den Verlust der Sammlung. Erst später erfuhr er, dass Teile der Sammlung in der DDR gefunden worden waren und sich in dem Museum für Deutsche Geschichte in Ost-Berlin befanden. 1974 verstarb Dr. Sachs und wurde von seiner Ehefrau beerbt. Diese starb 1998, ohne nach der Wiedervereinigung Ansprüche wegen der Sammlung geltend gemacht zu haben. Der Kläger ist ihr Erbe.

Die Plakatsammlung, von der zur Zeit 4.259 Plakate identifiziert sind, befindet sich heute im Besitz der Beklagten, einer Stiftung des öffentlichen Rechts. Mit der Klage hat der Kläger die Herausgabe zweier Plakate („Dogge“ und „Die blonde Venus") verlangt. Die Beklagte hat im Wege der Widerklage die
Feststellung beantragt, dass der Kläger nicht Eigentümer der Plakatsammlung ist, hilfsweise, dass er nicht berechtigt ist, die Plakate herauszuverlangen.

Das Landgericht (Entscheidung vom 10. Februar 2009 - 190 116/08 [Anmerkung der Redaktion: abgedruckt in KUR 2009, 57 ff.]) hat die Beklagte zur Herausgabe eines der Plakate („Dogge“) verurteilt und die weitergehende Klage sowie die Widerklage abgewiesen. Auf die von beiden Parteien eingelegte Berufung hat das Kammergericht (Entscheidung vom 28. Januar 2010 - 8 U 56/09 [Anmerkung der Redaktion: abgedruckt in KUR 2010, 17 ff.]) unter Abweisung aller übrigen Anträge der Widerklage im Hilfsantrag stattgegeben. Mit der von dem Senat zugelassenen Revision möchte der Kläger, der den Herausgabeanspruch hinsichtlich des zweiten Plakats („Die blonde Venus") nicht weiterverfolgt, die Verurteilung der Beklagten in dem durch das Landgericht zuerkannten Umfang sowie die vollständige Abweisung der Widerklage erreichen. Die Beklagte, die die Zurückweisung der Revision erstrebt, hat Anschlussrevision eingelegt, mit der sie sich gegen die Abweisung der Widerklage im Hauptantrag wendet. Der Kläger beantragt die Zurückweisung der Anschlussrevision.

\section{Entscheidungsgründe}

I.

Das Berufungsgericht, dessen Entscheidung in ZOV 2010, 87, veröffentlicht ist, meint, der Vater des Klägers habe sein Eigentum an der Plakatsammlung weder vor noch durch deren Wegnahme 1938 verloren. Ebenso wenig habe er das Eigentum im Rahmen des Wiedergutmachungsverfahrens verloren.

Die Plakate seien auch nicht in das Volkseigentum der DDR übergegangen. Dem danach an sich gegebenen Herausgabeanspruch des Klägers nach § 985 BGB stünden allerdings die Vorschriften des alliierten Rückerstattungsrechts und des Bundesrückerstattungsgesetzes entgegen. Es entspreche gefestigter höchstrichterlicher Rechtsprechung, dass Ansprüche wegen nationalsozialistischer Unrechtsakte nur nach Maßgabe der 
Rückerstattungs- und Entschädigungsgesetze geltend gemacht werden könnten. Darüber hinaus sei ein etwaiger Herausgabeanspruch des Klägers verwirkt.

Das hält einer rechtlichen Nachprüfung nicht in allen Punkten stand.

\section{II.}

\section{Revision des Klägers}

Die Revision des Klägers ist begründet. Zu Unrecht hat das Berufungsgericht einen Anspruch des Klägers auf Herausgabe des Plakats „Dogge“ gemäß § 985 BGB verneint und auf die Widerklage der Beklagten festgestellt, dass der Kläger nicht berechtigt ist, die vormals seinem Vater gehörende Plakatsammlung von der Beklagten herauszuverlangen.

1. Nach Auffassung des Berufungsgerichts ist der Kläger als Rechtsnachfolger (Erbeserbe) seines Vaters Eigentümer der Plakatsammlung. Das nimmt die Revision als für sie günstig hin (zur Anschlussrevision siehe unter III.).

2. Der Herausgabeanspruch nach $\S 985$ BGB wird nicht durch die besonderen Regelungen über die Wiedergutmachung des nationalsozialistischen Unrechts verdrängt.

a) Zutreffend geht das Berufungsgericht davon aus, dass der Anspruch nicht durch das Vermögensgesetz ausgeschlossen wird. Dieses findet zwar gemäß § 1 Abs. 6 VermG auch auf vermögensrechtliche Ansprüche von Bürgern Anwendung, die wie der Vater des Klägers in der Zeit vom 30. Januar 1933 bis zum 8. Mai 1945 aus rassischen Gründen verfolgt wurden und deshalb ihr Vermögen infolge von Zwangsverkäufen, Enteignungen oder auf andere Weise verloren haben. Die von dem Senat (Urteil vom 7. Juli 1995 - V ZR 243/94, BGHZ 130, 231 [235]) bislang nur für den Restitutionsanspruch nach $\S 1$ Abs. 1 Buchstabe $c$ und Abs. 3 VermG bejahte Frage, ob ein nach den vermögensrechtlichen Bestimmungen begründeter Anspruch einem zivilrechtlichen Anspruch vorgeht, der seinen Grund ebenfalls in dem von dem Vermögensgesetz erfassten staatlichen Unrecht hat, stellt sich hier schon deshalb nicht, weil der von dem Vater des Klägers erlittene Vermögensverlust keinen Restitutionsanspruch nach der Vorschrift in § 1 Abs. 6 VermG auslöst. Deren Anwendung setzt nämlich voraus, dass der Vermögenswert in dem Zeitpunkt der Schädigung im Beitrittsgebiet belegen war (vgl. BVerwGE 135, 272 [277] Rn. 31 mwN). Daran fehlt es hier, da die Plakatsammlung nach den Feststellungen des Berufungsgerichts in Berlin-Schöneberg und somit im späteren Westteil der Stadt beschlagnahmt wurde.

b) Entgegen der Auffassung des Berufungsgerichts tritt der Herausgabeanspruch nicht hinter die Vorschriften des alliierten Rückerstattungsrechts, hier die in Berlin geltende Anordnung BK/O (49) 180 der Alliierten Kommandantur Berlin betreffend die Rückerstattung feststellbarer Vermögensgegenstände an
Opfer der nationalsozialistischen Unterdrückungsmaßnahmen (vom 26. Juli 1949, VOBI. für Groß-Berlin I S. 221 - nachfolgend Rückerstattungsanordnung oder REAO) zurück.

aa) Allerdings hat der Bundesgerichtshof in ständiger Rechtsprechung entschieden, dass Ansprüche, die sich aus der Unrechtmäßigkeit einer nationalsozialistischen Enteignungsmaßnahme ergeben, grundsätzlich nur nach Maßgabe der zur Wiedergutmachung erlassenen Rückerstattungs- und Entschädigungsgesetze und in dem dort vorgesehenen Verfahren verfolgt werden können (vgl. Urteile vom 11. Februar 1953 II ZR 51/52, BGHZ 9, 34 [45]; vom 8. Oktober 1953 - IV ZR 30/53, BGHZ 10, 340 [343]; vom 5. Mai 1956 - VI ZR 138/54, RzW 1956, 237 sowie Beschluss vom 27. Mai 1954 - IV ZB 15/54, NJW 1954, 1368; ebenso die hM im älteren Schrifttum, vgl. Blessin/ Wilden Bundesrückerstattungsgesetz, 1958, Einl. Rn. 26; Goetze Die Rückerstattung in Westdeutschland und Berlin, 1950, Anm. zu Art. 57 REG [AmZ]; Harmening/Hartenstein/Osthoff Rückerstattungsgesetz, 2. Aufl., 1952, Einl. BI. Nr. 53 Rs.; Kubuschok/ Weißstein Rückerstattungsrecht, 1950, Art. 49 REG [BrZ] / Art. 57 REG [AmZ] Anm. 2; Muller Rückerstattung in Deutschland, 1948, Vorbem. S. 10; Korth SJZ 1948, 377, 383; aA van Dam Rückerstattungs-Gesetz für die Britische Zone, 1949, Einf. S. 15; von Godin Rückerstattung feststellbarer Vermögensgegenstände, 1950, Art. 57 REG [AmZ] Anm. 1; Dubro NJW 1953, 706).

Begründet wurde der Vorrang des Rückerstattungsverfahrens zum einen mit den besonderen Schwierigkeiten, die sich daraus ergaben, dass das geltende Recht keine ausreichende Grundlage bot, die durch die nationalsozialistischen Unrechtsmaßnahmen herbeigeführten Vermögensverschiebungen rückgängig zu machen (dazu ausführlich Anton Rechtshandbuch Kulturgüterschutz und Kunstrestitutionsrecht, Bd. 1, 2010, S. 689 ff.), und denen durch ein besonderes, die Ansprüche des Geschädigten abschließend regelndes Gesetz begegnet werden sollte. Zum anderen sollten durch die - im Vergleich zu den allgemeinen Verjährungsfristen deutlich kürzeren - Fristen, innerhalb deren ein Rückerstattungsanspruch durch den Geschädigten anzumelden war (nach Art. 50 Abs. 2 Satz 1 REAO bis zum 30. Juni 1950), das Interesse der Allgemeinheit an der baldigen Beruhigung des Wirtschaftslebens sowie das Interesse des Rückgewährpflichtigen geschützt werden, nach dem Fristablauf nicht mehr mit weiteren Ansprüchen des Geschädigten rechnen zu müssen (vgl. BGH, Urteil vom 8. Oktober 1953 - IV ZR 30/53, BGHZ 10, 340 [343 ff.]).

bb) Demgegenüber herrscht im neueren Schrifttum zum Teil im Anschluss an eine Entscheidung des Großen Senats für Zivilsachen (Beschluss vom 28. Februar 1955 - GSZ 4/54, BGHZ 16,350 ) die Auffassung vor, dass das Rückerstattungsrecht in erster Linie den Interessen des Geschädigten gedient habe. Das schließe es aus, dem Geschädigten Ansprüche zu versagen, die bereits nach den allgemeinen zivilrechtlichen Bestimmungen durch die Unrechtsmaßnahme begründet worden seien ( $\mathrm{vgl}$. Hartung Kunstraub in Krieg und Verfolgung, 2005, S. 169; Rudolph Restitution von Kunstwerken aus jüdischem Besitz, 2007, 
S. 94 ff.; Schulze Kunstrechtsspiegel 2010, 8 [9]; IPrax 2010, 290 [297]; Weller Kunstrechtsspiegel 2009, 32 [35 sowie 42, 43]; ähnlich bereits Mosheim BB 1949, 27: „MeistbegünstigungsPrinzip").

cc) Ob die zuletzt genannte Ansicht Veranlassung bietet, die bisherige Rechtsprechung in Frage zu stellen, kann dahinstehen. Den alliierten Rückerstattungsvorschriften kommt jedenfalls dann kein Vorrang gegenüber einem Herausgabeanspruch nach § 985 BGB zu, wenn der verfolgungsbedingt entzogene Vermögensgegenstand wie hier und anders als in den bislang durch den Bundesgerichtshof entschiedenen Fällen nach dem Krieg verschollen war und der Berechtigte erst nach Ablauf der für die Anmeldung eines Rückerstattungsanspruchs bestimmten Frist von seinem Verbleib Kenntnis erlangt hat.

Entgegen der Auffassung des Berufungsgerichts steht Art. 51 Satz 1 REAO der Geltendmachung eines Herausgabeanspruchs in einem solchen Fall nicht entgegen. Zwar können danach, soweit nichts anderes bestimmt ist, Ansprüche, die unter die Rückerstattungsanordnung fallen, nur nach deren Maßgabe und unter Einhaltung der darin geregelten Fristen geltend gemacht werden. Die nach der Rechtsprechung des Bundesgerichtshofs (vgl. Urteil vom 8. Oktober 1953 - IV ZR 30/53, BGHZ 10, 340 [344] für die vergleichbaren Regelungen in der Amerikanischen und der Britischen Zone) von der Vorschrift ausgehende Sperrwirkung wird indes durch den die Anordnung beherrschenden Grundsatz der Naturalrestitution begrenzt.

(1) Die Rückerstattungsanordnung regelt in erster Linie die Rückerstattung "feststellbarer“ Vermögensgegenstände (vgl. $\S 1$ Abs. 1 REAO). Der Begriff "feststellbar" („identifiable") diente ursprünglich in Entwurfsfassungen dazu, den Anwendungsbereich der Alliierten Anordnungen auf Rechtsverluste zu begrenzen, die durch Rückgabe des entzogenen Vermögensgegenstands in natura wiedergutgemacht werden konnten (vgl. ORG Nürnberg, RzW 1959, 371 [372 r. Sp.] sowie Schwarz Rückerstattung nach den Gesetzen der Alliierten Mächte, 1974, S. 118 f.). Von inm erfasst sind nur Gegenstände, zu deren Rückforderung sich der Berechtigte tatsächlich imstande sah, weil ihm die Person des gegenwärtigen Besitzers bekannt war (vgl. Art. 1 Abs. 2 REAO; Goetze aaO, Art. 1 REG [AmZ] Anm. 2; i. Erg. ebenso Harmening/Hartenstein/Osthoff aaO, Art. 1 REG [BrZ] Anm. III. 2). Diese Voraussetzung war bei einem Gegenstand, über dessen Existenz und Verbleib wie im Fall der dem Vater des Klägers gehörenden Plakatsammlung in dem Zeitraum, in dem ein Verfahren nach der Rückerstattungsanordnung eingeleitet werden konnte, Unklarheit herrschte, nicht erfüllt.

(2) Die Rückerstattungsanordnung sieht allerdings auch Ersatzansprüche des Berechtigten für den Fall vor, dass der Gegenstand bei dem Rückerstattungspflichtigen untergegangen oder diesem die Herausgabe aus sonstigen Gründen unmöglich war (Art. 26 Abs. 3 und Art. 27 Abs. 2 REAO). Bei dem Schadensausgleich in Geld handelte es sich nach der Vorstellung der Alliierten indes um eine nachrangige Form der Wiedergutmachung; in erster Linie hatte diese durch Rückgabe des entzogenen Vermögens an den Berechtigten zu erfolgen (vgl. Vorbemerkung sowie Art. 1 REAO; ebenso Art. 1 Abs. 1 REG [AmZ]; Art. 1 Abs. 1 REG [BrZ]; Art. 5 der Verordnung Nr. 120 [FrZ]; BGH Urteil vom 5. Mai 1956 - VI ZR 138/54, RzW 1956, 237 [238]; Blessin/Wilden aaO, Einl. Rn. 15; Schwarz aaO, S. 122 und S. 175). Dass die auf eine Ausgleichsleistung in Geld gerichteten Ansprüche aus der Rückerstattungsanordnung bei einer zunächst verschollenen, nach Ablauf der Anmeldefrist aber wieder aufgetauchten Sache dennoch als abschließende Wiedergutmachung anzusehen sein sollten, ergibt sich - ungeachtet einer in diesem Fall etwa bestehenden Pflicht, eine bereits empfangene Ausgleichszahlung zurückzuerstatten - aus der Rückerstattungsanordnung nicht (vgl. BVerwG ZIP 1997, 1392 [1393] zu dem Restitutionsanspruch nach $\S 1$ Abs. 6 VermG).

(3) Das vorrangige Ziel der Naturalrestitution steht ferner der Annahme entgegen, ein zivilrechtlicher Herausgabeanspruch werde durch die alliierte Rückerstattungsanordnung auch dann verdrängt, wenn es dem Berechtigten unmöglich war, die Rückgabe des entzogenen Vermögensgegenstands in deren Rahmen zu erreichen, weil dieser wie hier bis zum Ablauf der Anmeldefrist des $\S 50$ Abs. 2 REAO verschollen und damit nicht "feststellbar" war. Bliebe es in einem solchen Fall auch nach dem Wiederauffinden des Gegenstands bei der von dem Bundesgerichtshof bislang angenommenen Sperrwirkung des Art. 51 Satz 1 REAO, wären der Berechtigte und seine Rechtsnachfolger von der vorrangig angestrebten Wiedergutmachung durch Rückgabe dauerhaft ausgeschlossen, obwohl diese, wenn auch zu einem späteren Zeitpunkt, tatsächlich und auf der Grundlage der allgemeinen Gesetze auch rechtlich möglich ist. Die alliierten Rückerstattungsbestimmungen hätten dem Berechtigten damit jede Möglichkeit genommen, die Wiederherstellung des rechtmäßigen Zustands zu verlangen und auf diese Weise das nationalsozialistische Unrecht perpetuiert. Ein solches Ergebnis ist mit dem Sinn und Zweck dieser Bestimmungen, die Interessen des Geschädigten zu schützen (vgl. BGH Beschluss vom 28. Februar 1955 - GSZ 4/54, BGHZ 16, 350 [357]), nicht zu vereinbaren.

c) Auch das Bundesrückerstattungsgesetz steht dem Herausgabeanspruch des Klägers nicht entgegen. Denn es schuf lediglich eine gesetzliche Grundlage für die Berechnung und Erfüllung der bereits nach anderen Rechtsvorschriften entstandenen, auf einen Geldbetrag oder auf Schadensersatz gerichteten Rückerstattungsansprüche gegen das Deutsche Reich (vgl. § 2 iVm § 11 Nr. 1 BRüG; Biella Das Bundesrückerstattungsgesetz, 1981, S. 83 f.; Kemper/Burkhardt Bundesrückerstattungsgesetz, 2. Aufl., 1957, Einf. S. 16) und eröffnete insoweit die Anmeldefristen neu (vgl. § 29 BRüG). Bestimmungen, aus denen sich ergibt, dass die Rechte, die dem Berechtigten aufgrund des Eigentums an der (vermeintlich) untergegangenen Sache zustehen, mit der Erfüllung des Rückerstattungsanspruchs auf die öffentliche Hand übergehen, enthält es nicht. Ebenso wenig begründete es, von den hier nicht einschlägigen Vorschriften der $\S \S 12,13$ BRüG abgesehen, neue Ansprüche zugunsten der von einer 
nationalsozialistischen Verfolgungsmaßnahme Betroffenen, hinsichtlich deren sich die Frage nach dem Verhältnis zu den nach dem allgemeinen Zivilrecht gegebenen Ansprüchen stellen könnte.

3. Dass der Vater des Klägers, was dem Herausgabeanspruch entgegenstehen könnte, im Zusammenhang mit der ihm im Jahr 1961 gewährten Wiedergutmachung eine Erklärung abgegeben hat, in der er auf alle bestehenden Rechte wegen der Plakatsammlung verzichtet hat, ist durch das Berufungsgericht nicht festgestellt worden. Da ein Verzicht auf Rechte im Allgemeinen nicht zu vermuten ist, wäre ein unzweideutiges Verhalten erforderlich, das von dem Erklärungsgegner als Aufgabe des Rechts verstanden werden konnte (vgl. BGH Urteil vom 16. November 1993 - XI ZR 70/93, WM 1994, 13). Diese Voraussetzung ist durch das Schreiben des Vaters des Klägers aus dem Jahr 1966, in dem dieser gegenüber einem Mitarbeiter des Museums für Deutsche Geschichte in Ost-Berlin ausgeführt hat, er sei lediglich ideell und nicht materiell an einer Zusammenarbeit interessiert und habe im Übrigen eine größere Abfindungssumme erhalten, die alle seine Ansprüche abdecke, nicht erfüllt. Die Betonung des rein ideellen Interesses an der Sammlung durch den Vater des Klägers dürfte in erster Linie dazu gedient haben, die naheliegende Befürchtung des Museumsmitarbeiters auszuräumen, er werde Rechte wegen der Sammlung geltend machen, um so einen Kontaktabbruch des Museums zu vermeiden. Dabei ist zu berücksichtigen, dass dem Vater des Klägers ein Herausgabeverlangen gegenüber einem staatlichen Museum der DDR in den Zeiten des Kalten Krieges aussichtslos erscheinen musste; denn auch dies spricht dafür, dass mit dem Hinweis auf die erhaltene Entschädigung kein endgültiger Verzicht auf Rechte an der Sammlung zum Ausdruck gebracht, sondern etwaiges Misstrauen des Museums hinsichtlich des Grundes für die Kontaktaufnahme zerstreut werden sollte.

4. Der Herausgabeanspruch, hinsichtlich dessen die Beklagte die Einrede der Verjährung ausdrücklich nicht erhebt, ist nicht verwirkt.

a) Ein Recht ist verwirkt, wenn sich der Schuldner wegen der Untätigkeit seines Gläubigers über einen gewissen Zeitraum hin bei objektiver Beurteilung darauf einrichten darf und eingerichtet hat, dieser werde sein Recht nicht mehr geltend machen, und deswegen die spätere Geltendmachung gegen Treu und Glauben verstößt. Zu dem Zeitablauf müssen besondere, auf dem Verhalten des Berechtigten beruhende Umstände hinzutreten, die das Vertrauen des Verpflichteten rechtfertigen, der Berechtigte werde seinen Anspruch nicht mehr geltend machen (st. Rspr., vgl. Senat Urteile vom 12. Dezember 2008 - V ZR 49/08, NJW 2009, 847 [849] Rn. 39 [insoweit in BGHZ 179, 146 nicht abgedruckt] und vom 30. Oktober 2009 - V ZR 42/09, NJW 2010, 1074 [1076] Rn. 19 mwN). Verwirkung kann auch bei dem Herausgabeanspruch des Eigentümers nach § 985 BGB eintreten (vgl. Senat Urteil vom 30. April 1993 - V ZR 234/91, BGHZ 122, 308 [314] zu § 894 BGB). Dabei ist aber zu berücksichtigen, dass der Anspruch Kernbestandteil des Eigentums ist und seine
Verneinung wirtschaftlich die Enteignung des Eigentümers bedeutet, weshalb eine Verwirkung nur in Ausnahmefällen angenommen werden kann (vgl. Senat Urteil vom 16. März 2007 - V ZR 190/06, NJW 2007, 2183 [2184] mwN).

\section{b) Ein Fall der Verwirkung liegt hier nicht vor.}

aa) Entgegen der Auffassung des Berufungsgerichts kann die Zeit vor dem 3. Oktober 1990 für die Beurteilung, ob es sich bei der Geltendmachung des Herausgabeanspruchs durch den Kläger um eine unzulässige Rechtsausübung handelt, nicht berücksichtigt werden. Denn bis zu diesem Tag musste sich ein von dem Vater oder (nach dessen Tod im Jahr 1974) der Mutter des Klägers geäußertes Rückgabeverlangen, wovon auch das Berufungsgericht ausgeht, als offensichtlich aussichtslos erweisen, weil sich die Plakatsammlung auf dem Gebiet der DDR befand und daher ein privatrechtlicher Herausgabeanspruch aller Wahrscheinlichkeit nach nicht hätte durchgesetzt werden können (vgl. BGH Urteil vom 14. Januar 1964 - VI ZR 44/63, VersR 1964, 404 [405] zu dem „umgekehrten“ Fall, dass der Gläubiger des Anspruchs in der DDR ansässig war; Schoen NJW 2001, 537 [543]). Soweit das Berufungsgericht die Zeit bis zur Wiedervereinigung gleichwohl unter Hinweis auf die die Hemmung der Verjährung wegen höherer Gewalt betreffende Vorschrift des § 206 BGB für berücksichtigungsfähig hält, übersieht es, dass sich der Regelung keine über die Verjährung hinausgehenden Grundsätze entnehmen lassen (vgl. BGH Urteil vom 24. Oktober 1960 - III ZR 132/59, BGHZ 33, 360 [363]; Erman/Schmidt-Räntsch BGB, 13. Aufl., § 206 Rn. 2 mwN).

bb) Der danach maßgebliche Zeitraum von 16 Jahren, in dem die Mutter des Klägers sowie (nach deren Tod im Jahr 1998) der Kläger selbst von der Geltendmachung eines Herausgabeanspruchs abgesehen haben, ist für sich genommen nicht ausreichend, die Verwirkung des Anspruchs zu begründen (vgl. Senat Urteil vom 30. Oktober 2009 - V ZR 42/09, NJW 2010, 1074 [1075] Rn. 19). Zusätzliche Umstände, aus denen die Beklagte schließen durfte, ein Herausgabeanspruch wegen der Plakatsammlung werde nicht mehr geltend gemacht, sind nicht erkennbar. Der Inhalt des von dem Vater des Klägers verfassten Briefes aus dem Jahr 1966 (s.o. unter 3.) genügt für die Entstehung des für die Annahme der Verwirkung erforderlichen Vertrauenstatbestands ebenso wenig wie dessen Äußerung in einem 1970/71 veröffentlichten Artikel, wonach er sicher sei, dass "West- und Ostdeutschland (...) ihre Schätze zu hüten wissen“. Denn hieraus ergibt sich allenfalls, dass der zu dieser Zeit bereits hochbetagte Vater des Klägers selbst keine - zu der damaligen Zeit ohnehin nicht durchsetzbaren - Ansprüche mehr verfolgen würde, nicht aber, dass sich auch seine Erben mit einem dauerhaften Verbleib der Sammlung in einem Museum einverstanden zeigen würden. Äußerungen, die etwas Anderes nahe legen, hat das Berufungsgericht nicht festgestellt.

cc) Zu Unrecht meint das Berufungsgericht schließlich, die Beklagte habe mit Ablauf der in $\S 30$ a Abs. 1 Satz 1 VermG bestimmten Frist zur Geltendmachung von Rückübertragungs- 
ansprüchen nach dem Vermögensgesetz am 30. Juni 1993 darauf vertrauen dürfen, keinem Herausgabeanspruch des Eigentümers der Plakatsammlung mehr ausgesetzt zu werden. Das Vermögensgesetz findet, wovon das Berufungsgericht an anderer Stelle selbst ausgeht, in dem hier zu entscheidenden Fall, in dem das von dem NS-Regime beschlagnahmte Vermögen erst nach seiner Entziehung in das Beitrittsgebiet verbracht wurde, keine Anwendung (vgl. BVerwGE 135, 272 [277] Rn. 31 sowie oben unter 2. a). Dass seine Anwendbarkeit bis zu der Entscheidung durch das Bundesverwaltungsgericht im Jahr 2009 im Schrifttum unterschiedlich beurteilt wurde, begründet kein schutzwürdiges Vertrauen des unberechtigten Besitzers.

dd) Ob sich, wie der Kläger meint, die Beklagte als Stiftung des öffentlichen Rechts schon im Hinblick auf die im Anschluss an die Washingtoner Erklärung vom 3. Dezember 1998 abgegebene „Erklärung der Bundesregierung, der Länder und der kommunalen Spitzenverbände zur Auffindung und zur Rückgabe NS-verfolgungsbedingt entzogenen Kulturgutes, insbesondere aus jüdischem Besitz“ vom 14. Dezember 1999 (jew. abgedruckt bei Anton aaO, S. 736 f. [739 f.]), wonach die Erklärenden „in den verantwortlichen Gremien der Träger einschlägiger öffentlicher Einrichtungen darauf hinwirken (werden), dass Kulturgüter, die als NS-verfolgungsbedingt entzogen identifiziert und bestimmten Geschädigten zugeordnet werden können, nach individueller Prüfung den legitimierten früheren Eigentümern bzw. deren Erben zurückgegeben werden“, nicht auf den Einwand der Verwirkung des Herausgabeanspruchs berufen kann, bedarf hier keiner Entscheidung.

\section{III.}

\section{Anschlussrevision der Beklagten}

Die Anschlussrevision der Beklagten, mit der diese das fehlende Eigentum des Klägers an der Plakatsammlung festgestellt wissen will, ist unbegründet. Der Vater des Klägers ist zu Lebzeiten Eigentümer der Sammlung geblieben. Nach seinem Tod ist das Eigentum im Wege der Erbfolge zunächst auf seine Ehefrau und anschließend auf den Kläger übergegangen.

1. Dass die Plakatsammlung dem Vater des Klägers 1938 im Auftrag des Reichspropagandaministeriums weggenommen wurde, änderte an den bestehenden Eigentumsverhältnissen nichts. Nach den nicht angegriffenen Feststellungen des Berufungsgerichts handelte es sich bei dem Zugriff um eine Wegnahme ohne förmlichen Enteignungsakt. Eine rechtliche Grundlage für die Aneignung des Besitzes an der Plakatsammlung durch das Deutsche Reich ist auch nicht in der 11. Verordnung zum Reichsbürgergesetz zu sehen, durch die u.a. der Verfall jüdischen Vermögens angeordnet wurde. Denn diese Verordnung ist wegen ihres den Grunderfordernissen jeder rechtsstaatlichen Ordnung widersprechenden Unrechtsgehalts als von vornherein nichtig anzusehen und hat daher keine Rechts- wirkungen zu erzeugen vermocht (vgl. BGH Beschluss vom 28. Februar 1955 - GSZ 4/54, BGHZ 16, 350 [353 f.]; BVerfGE 23, 98 [106]; BVerwGE 98, 261 [263]).

2. Ohne Erfolg macht die Beklagte geltend, der Vater des Klägers sei zu dem Zeitpunkt der Wegnahme nicht mehr Eigentümer der Plakatsammlung gewesen, weil er diese zuvor an den Bankier Dr. Lenz veräußert habe. Da sich die Sammlung bis zuletzt in seinen Händen befand, kommt nur eine Übereignung nach § 930 BGB in Betracht; sie erforderte, dass der Vater des Klägers seinen Eigenbesitz an der Sammlung aufgegeben und auf Grund eines vereinbarten Besitzmittlungsverhältnisses (§ 868 BGB) dem Erwerber den Besitz vermittelt hat. Die Annahme des Berufungsgerichts, diese Voraussetzungen ließen sich nicht feststellen, ist frei von Rechtsfehlern.

a) Entgegen der Auffassung der Beklagten hat das Berufungsgericht die an ein Besitzkonstitut zu stellenden Anforderungen nicht verkannt. Die Vereinbarung eines Besitzmittlungsverhältnisses ersetzt die in § 929 Satz 1 BGB vorgesehene Übergabe der Sache. Diese Funktion steht einem Eigentumswechsel entgegen, bei dem der Wille des Veräußerers, die in seinem (unmittelbaren) Eigenbesitz befindliche Sache künftig für einen anderen zu besitzen, nicht in irgendeiner Form, und sei es nur gegenüber dem Erwerber (vgl. BGH Urteil vom 18. November 1963 - VIII ZR 198/62, NJW 1964, 398 f.), erkennbar zu Tage tritt. Eine dermaßen im Verborgenen bleibende Übertragung des Eigentums wäre mit dem das Sachenrecht beherrschenden, wenn auch in § 930 BGB zugunsten einer Erleichterung des Rechtsverkehrs mit beweglichen Sachen eingeschränkten (vgl. PWW/Prütting BGB, 6. Aufl., § 930 Rn. 1) Publizitätsgrundsatz nicht zu vereinbaren.

b) Dafür, dass vor der Wegnahme der Plakatsammlung entweder durch die ausdrückliche Begründung eines Besitzkonstituts oder zumindest durch ein konkludentes Verhalten (vgl. BGH Urteil vom 29. Oktober 2001 - II ZR 314/99, NJW-RR 2002, 854 [855]; Palandt/Bassenge BGB, 71. Aufl., § 930 Rn. 8 mwN) die Änderung der vormaligen Besitzverhältnisse dokumentiert wurde, hat das Berufungsgericht nichts festzustellen vermocht. Die von der Beklagten angeführte Äußerung des Vaters des Klägers aus dem Jahr 1953, wonach die Sammlung zu dem Zeitpunkt ihrer Wegnahme bereits "förmlich übereignet" gewesen sei, lässt nicht den Schluss auf eine wirksame Eigentumsübertragung zu. Gleiches gilt für die Erklärung des Dr. Lenz aus dem Jahr 1946, nach der ihm die Sammlung „als Pfand“ übereignet worden sei, um sie auf diese Weise vor der drohenden Konfiszierung zu retten. Beide Äußerungen beschränken sich letztlich auf die Mitteilung einer mit Blick auf die Erklärung des Dr. Lenz zudem nicht eindeutigen Rechtsauffassung.

Ob diese zutrifft, kann in Ermangelung tatsächlicher Feststellungen zu dem zugrunde liegenden Geschehen nicht beurteilt werden. 
3. Ebenfalls ohne Erfolg macht die Beklagte geltend, das Eigentum an der Plakatsammlung sei dadurch, dass der Vater des Klägers diese nicht zur Rückerstattung angemeldet habe, kraft Gesetzes auf deren damaligen Besitzer übergegangen. Die Rückerstattungsanordnung hatte den Zweck, die beschleunigte Rückerstattung feststellbarer Vermögensgegenstände sicherzustellen (vgl. BGH Beschluss vom 28. Februar 1955 - GSZ 4/54, BGHZ 16, 350 [360]).

Konnten die durch die Anordnung begründeten Ansprüche aufgrund des Ausschlusscharakters der Anmeldefrist für sie nicht mehr durchgesetzt werden, musste derjenige, der den Gegenstand damals im Besitz hatte, zwar nicht mehr damit rechnen, Rückerstattungsansprüchen ausgesetzt zu sein. Ein originärer Eigentumserwerb durch Rückerstattungspflichtige, die lediglich den Besitz, nicht aber das Eigentum an dem entzogenen Gegenstand erlangt hatten, war hiermit aber nicht verbunden.
4. Gegen die Annahme des Berufungsgerichts, der Vater des Klägers habe das Eigentum an der Plakatsammlung auch nicht zu einem späteren Zeitpunkt eingebüßt, erhebt die Beklagte keine Einwände. Rechtsfehler sind insoweit auch nicht ersichtlich.

\section{IV.}

Das Berufungsurteil ist somit in dem durch die Revision angefochtenen Umfang aufzuheben (§562 Abs. 1 ZPO). Der Senat hat in der Sache selbst zu entscheiden, weil die Aufhebung des Urteils nur wegen einer Rechtsverletzung bei der Anwendung des Gesetzes erfolgt und nach letzterem die Sache zur Endentscheidung reif ist (§563 Abs. 3 ZPO).

V.

Die Kostenentscheidung beruht auf § 91 Abs. 1, § 92 Abs. 2, § 97 Abs. 1 ZPO. (Entscheidung von der Redaktion bearbeitet.)

\section{Beweislastverteilung bei vermögensrechtlichen Rückgabeansprüchen}

Bundesverwaltungsgericht, Beschluss vom 7. März 2012 - 5 B 56/11

\section{Zu Fragen der Beweislast und möglichen Beweiserleichterungen in einem Rechtsstreit um die Rückgabe eines} Bildes nach § 5 Abs. 1 Satz 1 AusglLeistG. (Leitsatz der Redaktion)

\section{Tenor}

- Die Beschwerde der Kläger gegen die Nichtzulassung der Revision in dem Urteil des Verwaltungsgerichts Gera vom 21. Juni 2011 wird zurückgewiesen.

Die Kläger tragen die Kosten des Beschwerdeverfahrens mit Ausnahme der außergerichtlichen Kosten der Beigeladenen.

Der Wert des Streitgegenstandes für das Beschwerdeverfahren wird auf 5.000,- EUR festgesetzt.

\section{Gründe}

Die Beschwerde hat keinen Erfolg. Die geltend gemachten Zulassungsgründe des § $132 \mathrm{Abs} .2 \mathrm{Nr}$. 1 und 3 VwGO liegen nicht vor.

1. Die Zulassung der Revision ist nicht wegen grundsätzlicher Bedeutung der angesprochenen Beweisfragen geboten ( $§ 32$ Abs. 2 Nr. 1 VwGO). Grundsätzliche Bedeutung kommt einer Rechtssache zu, wenn für die angefochtene Entscheidung der Vorinstanz eine konkrete, fallübergreifende und bislang höchstrichterlich ungeklärte Rechtsfrage des revisiblen Rechts von Bedeutung war, deren Klärung im Revisionsverfahren zu erwarten und zur Erhaltung der Einheitlichkeit der Rechtsprechung oder zur Weiterentwicklung des Rechts geboten ist (st. Rspr., vgl. z.B. Beschluss vom 2. Februar 2011 - BVerwG 6 B 37.10 - Buchholz
421.2 Hochschulrecht Nr. 173). Diese Voraussetzungen liegen bei den von den Klägern aufgeworfenen Beweisfragen entweder nicht vor oder sind nicht ausreichend dargelegt ( $\$ 133$ Abs. 3 Satz 3 VwGO).

a) Das Verwaltungsgericht hat im vorliegenden Rechtsstreit um die Rückgabe eines Bildes nach $\S 5$ Abs. 1 Satz 1 AusglLeistG den Klägern die materielle Beweislast dafür auferlegt, dass diese Bilder bei der besatzungshoheitlichen Enteignung im September 1945 noch Einrichtungsgegenstände des Rittergutes gewesen und nicht schon vorher abhandengekommen sind. Die Kläger halten es für grundsätzlich klärungsbedürftig, ob das Verwaltungsgericht ihre unverschuldete Beweisnot in Bezug auf diese Frage nach dem zum Häftlings-, Vertriebenenund Asylrecht entwickelten Grundsätzen, nach den im Versicherungsrecht für den Fall des Diebstahls entwickelten Prinzipien oder durch eine Reduzierung des Beweismaßes hätte Rechnung tragen müssen (S. 8 und 10 der Beschwerdebegründung).

Diese Fragen bedürfen jedoch keiner rechtsgrundsätzlichen Klärung. Das Bundesverwaltungsgericht hat in Bezug auf vermögensrechtliche Rückgabeansprüche bereits mehrfach entschieden, dass auch im Vermögensrecht die Unerweislichkeit von Tatsachen, aus denen eine Partei ihr günstige Rechtsfolgen herleitet, grundsätzlich zu ihren Lasten geht (Urteil vom 24. März 1994 - BVerwG 7 C 11.93 - BVerwGE 95, 289 [294]; Beschluss vom 17. Mai 2005 - BVerwG 7 B 140.04 - [...] Rn. 6). In bestimmten typischen Sachverhaltskonstellationen - etwa bei Veräußerungen 